\title{
Possible role of Helicobacter pylori serology in reducing endoscopy workload
}

\author{
T.C.K. Tham, N. McLaughlin', D.F. Hughes ${ }^{2}$, M. Ferguson'1, J.J. Crosbie ${ }^{2}$, \\ M. Madden ${ }^{2}$, S. Namnyak ${ }^{1}$ and F.A. O'Connor
}

Departments of Medicine, ${ }^{1}$ Microbiology and ${ }^{2}$ Pathology, Atnagelvin Area Hospital, Londonderry, UK

\begin{abstract}
Summary: We validated a commercial enzyme-linked immunosorbent assay (ELISA), Helico-G, in diagnosing $H$. pylori in 129 patients (mean age 50 years, range 15-86). We analysed the results of endoscopy against serology to see whether there was a possibility of adopting the strategy of not endoscoping dyspeptic subjects under the age of 45. $\mathrm{H}$. pylori infection was considered present if either histology and/or culture were positive. The ELISA had a sensitivity of $88 \%$, specificity of $72 \%$, positive predictive value of $85 \%$, negative predictive value of $77 \%$ and accuracy of $82 \%$ in detecting $\mathrm{H}$. pylori. In a subgroup of 52 subjects aged 45 or less (mean age 35 years, range 15-45), 17 out of 25 patients with positive endoscopic findings were $H$. pylori seropositive while 16 out of 27 patients had normal endoscopic findings. Eighteen out of the 52 patients (35\%) were $\boldsymbol{H}$. pylori seronegative and normal endoscopically except for five patients $(\mathbf{1 0} \%)$ who had mild to moderate oesophagitis and two who had non-erosive gastritis (4\%). All patients with duodenal ulcer disease (7) were seropositive giving predictive values of positive and negative serology for a diagnosis of duodenal ulcer disease as $28 \%$ and $100 \%$, respectively.

Therefore adopting a strategy of endoscoping subjects under the age of $\mathbf{4 5}$ only if they were $\mathrm{H}$. pylori seropositive would have saved $35 \%$ of endoscopies in this age group but missed oesophagitis in $10 \%$. Negative serology would tend to exclude duodenal ulcer disease while positive serology discriminates poorly for it. Serology may be a useful adjunct in screening to reduce endoscopy workload provided that patients with gastro-oesophageal reflux symptoms are excluded.
\end{abstract}

\section{Introduction}

The serological tests for Helicobacter pylori that have been developed recently have many uses in epidemiology and other research, ${ }^{1}$ but their role in clinical practice is still under evaluation. Dyspepsia is a very common symptom in the community ${ }^{2}$ and its investigation constitutes a major workload for both radiology and endoscopy services. Most centres have experienced a steady increase in the yearly number of gastrointestinal endoscopies over the past decade. ${ }^{3}$ Investigating all dyspeptic patients by endoscopy would result in a low diagnostic yield; up to $75 \%$ of patient attending gastroenterology clinics with upper abdominal pain are diagnosed as having non-ulcer dyspepsia. ${ }^{4}$ Only about $20 \%$ of investigated patients prove to have peptide ulcer disease and $1-2 \%$ have gastric cancer. $^{3}$

As $H$. pylori infection of the stomach is strongly associated with peptic ulcers, and duodenal ulcer disease in particular, it has been suggested that

Correspondence: T.C.K. Tham, M.D., M.R.C.P., Department of Medicine, Institute of Clinical Science, Royal Victoria Hospital, Grosvenor Road, Belfast, UK. Accepted: 31 May 1994. noninvasive tests for the presence of the organism could be used as an adjunct or an alternative to endoscopy in diagnosing dyspepsia. There are two methods by which $H$. pylori can be detected noninvasively: serology and carbon-labelled urea breath tests. Serological tests have the advantage over carbon-labelled urea breath tests as they are cheap and simple to use, requiring just a blood sample. Thus serological screening for $H$. pylori antibodies has been suggested as a method to reduce endoscopy workload by excluding seronegative patients from endoscopy, as they would be less likely to have significant pathology. In order to avoid missing gastric carcinoma, an upper age limit of 45 years is imposed on those to be screened as gastric carcinoma is extremely rare in this age group (at least in the UK) constituting only $1.8 \%$ of cases. ${ }^{5}$

Our aims were: (1) to validate a commercial enzyme-linked immunosorbant assay (ELISA), Helico-G (Porton Cambridge, UK), against the currently accepted standards of histology, culture and urease tests for diagnosing the presence of $H$. pylori; and (2) to analyse the results of endoscopy against serology to see whether there was a possibility of adopting the strategy of not endoscoping 
subjects under the age of 45 who were $H$. pylori negative by serology.

\section{Methods}

Ethical approval for the study was granted by the Altnagelvin Medical Executive Committee and all patients gave written informed consent. Patients fasted for at least $\mathbf{8}$ hours prior to an endoscopy, which was performed with the Fujinon Electronic Video Endoscopy system. Before reuse, the endoscope and the biopsy forceps were washed, cleaned and soaked in glutaraldehyde solution for 10 minutes and thoroughly washed again with sterilized water.

A total of 129 consecutive patients (mean age 50 years, range 15-86) undergoing diagnostic endoscopy for dyspepsia, and a few other reasons such as chest pain and anaemia were tested for $H$. pylori antibodies by ELISA using an acid glycine extract of the bacterium (Helico-G, Porton Cambridge) to validate the test. From this group, all 52 subjects with dyspepsia aged 45 or less (mean age 35 years, range 15-45) were analysed for possible utility of $H$. pylori serological screening to reduce enduscopy workload. Two antral biopsy specimens were taken for histology (organism detected using cresyl fast violet stains), two for culture (immediate transfer to laboratory, Columbia blood agar with $H$. pylori selective supplement, micro-aerophilic conditions, $37^{\circ} \mathrm{C}, 5-7$ days) and one for urease (CLO, Delta West, Western Australia) tests. The activity of gastritis (normal, mild, severe) and degree of colonization with $H$. pylori (none, few, plenty of bacteria) were assessed semiquantitatively. ELISA analysis, histological examination and culture were performed independently without knowledge of endoscopic diagnosis. $H$. pylori was considered present when either histology or culture, or both were positive. The ELISA was considered seropositive for $H$. pylori infection if the antibody level was greater than 10 units $/ \mathrm{ml}$.

The Kruskall-Wallis non-parametric test followed by Mann-Whitney $U$-test was used to compare antibody levels of $H$. pylori with activity of gastritis and degree of $\boldsymbol{H}$. pylori colonization. $P<0.05$ was considered significant.

\section{Results}

A total of 73 out of 83 patients colonized with $H$. pylori were seropositive and 33 out of 46 patients not colonized with $H$. pylori were seronegative (Table I). Thus the ELISA had a sensitivity of $88 \%$ (95\% confidence limits $76-92 \%)$, specificity of $72 \%(57-84)$, positive predictive value of $85 \%$ (76-92), negative predictive value of $77 \%(61-68)$

and accuracy of $82 \%(75-88)$ for $H$. pylori infec-z tion. A total of 71 out of 83 patients colonized with $\frac{\mathbb{\Phi}}{\alpha}$ $H$. pylori were CLO test positive and 45 out of $46 c$. patients not colonized with $H$. pylori were CLO test $\Rightarrow$ negative (Table II). Thus in comparison, the CLO test had a sensitivity of $86 \%$ (95\% confidence limits? 76-92), specificity of $98 \%(88-99.9)$, positive predictive value of $99 \%(93-99.9)$, negative predic- $\frac{}{5}$ tive value of $79 \%(66-88)$ and accuracy of $91 \% \stackrel{\circ}{\circ}$ (83-95).

The median (interquartile range) antibody levelsof $H$. pylori from the ELISA increased significantly: in relation to the activity of gastritis $(P<0.05), \vec{\omega}$ with $6(4-7), 38(14-70)$ and $76(38-93)$ units/ml for normal, mild and severe gastritis, respectively: The median (interquartile range) antibody levels of $H$. pylori from the ELISA for few and plenty of $O$ bacteria were significantly increased when compared to no bacteria $(P<0.05)$, and showed ac trend to increase when compared to each othero $(P>0.05)$ : 7 (5-18), 47 (23-83), 69 (39-93) U/ml ${ }^{\odot}$ for none, few and plenty of bacteria, respectively..

In the 52 patients aged 45 or less, 17 out of $25^{-}$ patients with positive endoscopic findings were $H$.O

Table I Comparison of ELISA for the detection of $H$. ำ pylori (positive indicating presence of $\boldsymbol{H}$. pylori) woh histology and culture $\left(H\right.$. pylori being considered present $t_{0}$ if histology and/or culture were positive) in 129 patien.

\begin{tabular}{lccl}
\hline & ELISA positive & ELISA negative \\
\hline$H$. pylori present & 73 & 10 & pylori absent \\
$H .0$
\end{tabular}

\begin{tabular}{|c|c|}
\hline & (95\% CI intervals) \\
\hline Sensitivity & $88 \%(76-92)$ \\
\hline Specificity & $72 \%(57-84)$ \\
\hline Positive predictive value & $85 \%(76-92)$ \\
\hline Negative predictive value & $77 \%(61-88)$ \\
\hline Accuracy & $82 \%(75-88)$ \\
\hline
\end{tabular}

Table II Comparison of CLO for the detection of $H$. 을 pylori (positive indicating presence of $\boldsymbol{H}$. pylori) with histology and culture ( $H$. pylori being considered present음 if histology and/or culture were positive) in 129 patients

\begin{tabular}{lccc}
\hline & CLO positive & CLO negative & 三 \\
\hline H. pylori present & 71 & 12 & ○ \\
H. pylori absent & 1 & 45 & N \\
\hline
\end{tabular}

\begin{tabular}{|c|c|}
\hline & (95\% CI intervals) \\
\hline Sensitivity & $86 \%(76-92)$ \\
\hline Specificity & $98 \%(88-99.9)$ \\
\hline Positive predictive value & $99 \%(93-99.9)$ \\
\hline Negative predictive value & $79 \%(66-88)$ \\
\hline Accuracy & $91 \%(83-95)$ \\
\hline
\end{tabular}


pylori seropositive while 11 out of 27 patients with normal endoscopic findings were $H$. pylori seronegative. Out of the patients with positive endoscopic findings, 10 had oesophagitis, one had a duodenal ulcer, six had duodenitis (presence of significant erythema, friability, oedema or erosions), five had gastritis, one had Barrett's oesophagus, one had Mallory-Weiss tear and one had oesophageal candidiasis. A total of 18 out of 52 patients $(35 \%)$ were $H$. pylori seronegative and out of these, five $(10 \%)$ had mild to moderate oesophagitis and two (4\%) had non-erosive gastritis. Duodenal ulcer disease is defined as the presence of a duodenal ulcer or duodenitis. Seven out of 25 seropositive patients had duodenal ulcer disease while all 27 who were seronegative did not have it. The predictive values of positive and negative serology for a diagnosis of duodenal ulcer disease were $28 \%$ and $100 \%$, respectively (Table III).

\section{Discussion}

Our results suggest that the Helico-G ELISA kit has acceptable sensitivity, specificity, positive and negative predictive values and accuracy for the noninvasive detection of $H$. pylori. These are comparable with findings by other investigators with sensitivities ranging from $79 \%$ to $91 \%$ and specificities ranging from $47 \%$ to $91 \%,{ }^{6-9}$ and also to other ELISA kits. ${ }^{6,8}$ Compared to the CLO test, the latter shows higher specificity, positive predictive value and accuracy, but has the disadvantage of being invasive; these figures being consistent with those found by Dill et al. ${ }^{10}$

We assessed a widely suggested strategy of using $H$. pylori serology as a screening procedure to reduce endoscopy workload. Patients with dyspepsia who were found to be $H$. pylori seropositive would go on to endoscopy to determine whether they had peptic ulcer disease, while negatives would be reassured and treated for non-ulcer dyspepsia. To avoid missing early gastric carcinoma, an upper age limit of 45 years would be used as discussed in the introduction. The assumption is that the chief aim of endoscopy in most patients is to detect ulcer disease rather than minor pathologies.

Table III Serological results and predictive values in all dyspeptic patients with and without duodenal ulcer (DU) disease (duodenal ulcer or duodenitis)

ELISA positive ELISA negative

\begin{tabular}{lrr}
\hline DU disease present & 7 & 0 \\
DU disease absent & 18 & 27 \\
\hline
\end{tabular}

The predictive values of positive and negative serology for a diagonsis of duodenal ulcer disease were 28 and $100 \%$, respectively.
We found that if this strategy had been employed, $35 \%$ of endoscopies would have been saved and we did not miss patients with duodenal ulcer disease; duodenitis is considered as part of the spectrum of duodenal ulcer disease consistent with current opinion. ${ }^{11}$ The strategy detected all the seven $(13 \%)$ patients with duodenal ulcer disease. The high negative predictive value of the serology for duodenal ulcer disease suggests that negative serology would probably exclude duodenal ulcer disease, while its low positive predictive value for this suggests that it discriminates poorly for it. Our study supports the findings of Collins et al.,$^{12}$ although they used an in-house non-commercial ELISA.

However, using this strategy, oesophagitis $(10 \%)$ and gastritis $(4 \%)$ would have been missed. The clinical relevance of gastritis is uncertain. The presence of oesophagitis is probably clinically significant. To avoid missing this, a patient with a history suggestive of oeosphageal reflux may be excluded from this strategy to be endoscoped regardless of serology results as there is good correlation between the symptoms of gastro-oesophageal reflux disease and endoscopic findings. ${ }^{13}$ In addition the value of endoscopy in managing this type of patient is arguable. Endoscopy is not the gold standard for the diagnosis of reflux disease $\mathrm{e}^{14,15}$ and, if serology can act as a tool in the diagnostic process by excluding concurrent peptic ulcer disease, then the indications for endoscopy are diminished. The reason that $H$. pylori serology is probably of little help in screening for oesophagitis is because the association between the condition and $H$. pylori is at present uncertain. ${ }^{16}$

It is presently not known what happens to the patients who are seronegative and hence not endoscoped. The total number of endoscopies being performed may not be saved if these patients end up having an endoscopy anyway perhaps after a brief therapeutic tussle in the outpatient clinic. A prospective study of this group of patients is required if endoscopy saving is to be confirmed. As serological tests have a high sensitivity for $H$. pylori, the peptic ulcers missed will be $H$. pylori negative ulcers. The clinical importance of missing such a diagnosis is uncertain as it is not known whether they follow a different clinical course to $H$. pylori-positive peptic ulcers or not.

In conclusion, the Helico-G ELISA provides a useful noninvasive serodiagnostic test for $H$. pylori comparing favourably with current established standards. It may be useful as part of a screening programme to reduce endoscopy workload provided that patients with symptoms suggestive of gastro-oesophageal reflux are excluded from this. However, further larger prospective studies would be required to confirm this. 


\section{Acknowledgements}

We wish to thank Vector Scientific Ltd, Belfast, for funding the study and the nursing staff in the Day Case Unit of the hospital for their cooperation.

\section{References}

1. Newell, D.G. \& Stacey, A.R. The serology of Helicobacter pylori infections. In: Rathbone, B.J. \& Heatley, R.V. (eds). Helicobacter pylori and Gastroduodenal Disease, 2nd edn. Blackwell Scientific Publications, Oxford, 1992, pp. 64-73.

2. Jones, R. \& Lydeard, S. Prevalence of symptoms of dyspepsia in the community. Lancet 1989, 1: 47-51.

3. Sobala, G.M. Possible clinical uses of serology of Helicobacter pylori. In: Malfertheiner, P. \& Ditschuneit, H. (eds) Helicobacter pylori, Gastritis and Peptic Ulcer. SpringerVerlag, Berlin, 1990, pp. 147-153.

4. Gear, M.W.L. \& Barnes, R.J. Endoscopic studies of dyspepsia in a general practice. $\mathrm{Br}$ Med $J$ 1980, 280: 1136-1137.

5. Williams, B., Luckas, M., Ellingham, J.H.M., Dain, A. \& Wicks, A.C.B. Do young patients with dyspepsia need investigation? Lancet 1988, 2: 1349-1351.

6. Yamamoto, I., Fukuda, Y., Tonokatsu, Y. et al. Comparison of serodiagnostic commercial kit for Helicobacter pylori detection using sera in Japan. Ir J Med Sci 1992, 161 (Suppl 10): 75.

7. Marks, J., Gopal Rao, G., Cobden, I., Johri, R., Johri, S. \& Rodgers, A.D. Evaluation of ELISA in diagnosis of Helicobacter pylori infection. Ir J Med Sci 1992, 161 (Suppl 10): 80.

8. Tahar, A.S., Reid, J., Boothmann, P. et al. Serological diagnosis of Helicobacter pylori - evaluation of four tests in the presence or absence of non-steroidal anti-inflammatory drugs. Ir J Med Sci 1992, 161 (Suppl 10): 81.

9. Mendall, M.A., Goggin, P.M., Molineaux, N.J. et al. $H$. pylori serology reduces the workload from direct access endoscopy. Clin Sci 1992, 82 (Suppl): 24P.
10. Dill, S., Payne-James, J.J., Misiewicz, J.J. et al. Evaluation of ڤి ${ }^{13} \mathrm{C}$-urea breath test in the detection of Helicobacter pylori and in monitoring the effect of tripotassium dicitratobismuthate in non-ulcer dyspepsia. Gut 1990, 31: 1237-1241.

11. Misiewicz, J.J. \& Pounder, R.E. Peptic ulcer. In: Weatherall, D.J., Ledingham, J.G.G. \& Warrell, D.A. (eds) Oxford Textbook of Medicine, 2nd edn. Oxford University Press, Oxford, 1987, pp. 12.64-12.77.

12. Collins, J.S.A., Bamford, K.B., Sloan, J.M., Collins, B.J., Moorehead, R.J. \& Love, A.H.G. Screening for Helicobacter pylori antibody could reduce endoscopy workload in young dyspeptic patients. Eur J Gastroenterol Hepatol 1992, 4: 991-993.

13. Klauser, A.G., Schindlbeck, N.E. \& Muller-Lissner, S.A. Symptoms in gastro-oesophageal reflux disease. Lancet 1990, 335: 205-208.

14. Johansson, K.E., Ask, P., Boeryd, B., Fransson, S.-G. \& Tibbling, L. Oesophagitis, signs of reflux, and gastric secretion in patients with symptoms of gastro-oesophageal reflux disease. Scand J Gastroenterol 1986, 21: 837-847.

15. Knill-Jones, R.P., Card, W.I., Crean, G.P., James, W.B. \& Spiegelhalter, D.J. The symptoms of gastro-oesophageal 믐 reflux and oesophagitis. Scand $J$ Gastroenterol 1984, (Suppl 106): 72-76.

16. Cheng, E.H., Bermanski, P., Silversmith, M., Valenstein, P. Kawanishi, H. Prevalence of Campylobacter pylori in oesor phagitis, gastritis and duodenal disease. Arch Intern Med 1989, 149: 1373-1375. 